\title{
WEAKLY COMPACT GROUPS OF OPERATORS
}

\author{
T. A. GILLESPIE AND T. T. WEST
}

\begin{abstract}
It is shown that the weakly closed algebra generated by a weakly compact group of operators on a Banach space is reflexive and equals its second commutant. Also, an example is given to show that the generator of a monothetic weakly compact group of operators need not have a logarithm in the algebra of all bounded linear operators on the underlying space.
\end{abstract}

Let $X$ be a complex Banach space, $B(X)$ the algebra of all bounded linear operators on $X$, and $I$ the identity operator on $X$. By a group in $B(X)$ we shall mean a multiplicative group with unit $l$. The weak operator topology on $B(X)$ is denoted by the letter $w$. Given a nonempty subset $\mathcal{E}$ of $B(X), \mathcal{E}^{\prime}$ and $\mathcal{E}^{\prime \prime}$ denote the first and second commutants of $\mathcal{E}$, and $A(\mathcal{E})$ is the $w$-closed subalgebra of $B(X)$ generated by $\mathcal{E}$ and $I$. The lattice of all $\mathcal{E}$-invariant closed subspaces of $X$ is denoted by Lat $\mathcal{E}$, and

$$
\text { Alg Lat } \mathcal{E}=\{T \in B(X): T(L) \subset L(L \in \text { Lat } \mathscr{E})\} \text {. }
$$

A subalgebra $A$ of $B(X)$ is reflexive if $A \lg$ Lat $A=A$. It is clear that reflexive algebras are $w$-closed and contain $I$. Finally, $\mathbf{C}, \mathbf{R}, \mathbf{Z}$ and $\mathbf{T}$ are the complex numbers, the reals, the integers and the unit circle.

We present several results concerning $w$-compact groups in $B(X)$. Such groups come within the general framew ork discussed by de Leeuw in [1], where the underlying space is called a $G$-space. The monothetic (singly generated) case has been considered in [4], [5], where an operator in $B(X)$ generating a $w$-compact group (with unit $I$ ) is called a $G$-operator. It was shown in [4] that, if $\mathcal{G}$ is a monothetic $w$-compact group, then $A(\mathcal{G})$ is reflexive and $\mathcal{G}^{\prime \prime}=A(\mathcal{Y})$. In fact the methods developed there and in [5] can be extended to prove

Theorem 1. Let $\mathcal{G}$ be an abelian w-compact group in $B(X)$ (with unit I). Then $A(\mathcal{G})$ is reflexive and $\mathcal{G}^{\prime \prime}=A(\mathcal{G})$.

Received by the editors February 4, 1974.

AMS (MOS) subject classifications (1970). Primary 47D10, 47A15, 46L20; Secondary 43A22, 43A05. 
Problem A. Does Theorem 1 remain valid if the hypothesis that $\mathcal{G}$ be abelian is omitted?

$G$-operators have occurred in the work of Ljubic [8], where a study is made of the spectral properties of an operator $S \in B(X)$ satisfying $\|\exp (i r S)\| \leq M(r \in \mathbf{R})$ and a certain almost-periodic condition. (The space $X$ is taken to be weakly sequentially complete.) It is shown that each such $S$ has a total set of eigenvectors corresponding to real eigenvalues, from which it is easily seen (via [4, Theorem 1.2]) that $\exp (i S)$ is a G-operator.

Problem B. Can every $G$-operator be written as $\exp (i S)$ for some bounded $S$ ?

Solution A. Theorem 1 does indeed extend to the nonabelian case and we sketch the main ideas of the proof.

Let $\oint$ be a $w$-compact group in $B(X)$.

Lemma 2 [2, Theorem 8.1]. $X$ is the closed linear span of finite dimensional 乌-invariant subspaces.

An easy consequence of this is the following description of Lat $\mathrm{S}_{\text {(cf. }}$ Corollary 1.4 of [4]).

Lemma 3. Each subspace in Lat $\mathrm{Y}$ is spanned by finite dimensional 乌-irreducible subspaces.

Write $X^{(n)}$ for the direct sum of $n$ copies of $X$ and $T^{(n)} \in B\left(X^{(n)}\right)$ for the $n$th direct sum of $T$. Putting $\mathcal{G}^{(n)}=\left\{T^{(n)}: T \in \mathcal{G}_{\}}\right.$, it is easy to see that $\mathcal{G}^{(n)}$ is a $w$-compact group in $B\left(X^{(n)}\right)$ with unit $I^{(n)}$.

Lemma 4. Let $S \in A \operatorname{Alg}$ Lat . Then $^{(n)} \in \operatorname{Alg}$ Lat $乌^{(n)}$ for $n=1,2, \ldots$.

This is the key result and we sketch its proof. A straightforward argument reduces the proof to the case $n=2$. It is then sufficient, by Lemma 3 , to show that each finite dimensional $S^{(2)}$-irreducible subspace $M$ of $X^{(2)}$ is $S^{(2)}$-invariant. Using irreducibility, this is easily done in the case when $M$ contains $(0, x)$ for some $x \neq 0$. Suppose therefore that $M$ contains no elements of this form. Then there is a finite dimensional subspace $N$ of $X$ and a linear operator $U: N \rightarrow X$ such that $M=\{(x, U x): x \in N\}$. The hypotheses on $M$ imply that $N$ and $U N$ belong to Lat $\varrho$ and that $(U T-T U)(N)=$ $\{0\}(T \in \mathcal{Y})$. Also, $N$ is $S_{\text {-irreducible and thus either }}$

(i) $U(N)=N$, or

(ii) $U(N) \cap N=\{0\}$. 
In case (i), $U$ commutes with the irreducible set of operators $\varrho_{\mid} \mid N$ on $N$. Hence $U$ is a scalar, from which it follows that $M \in$ Lat $S^{(2)}$. In case (ii), the subspace $L=(I+U) N$ is $\mathcal{G}$-and hence $S$-invariant. Therefore, given $x \in N$, there exists $y \in N$ such that $S x-y=-S U x+U y$. The lefthand side of this equation is in $N$ and the right in $U(N)$, since $N$ and $U(N)$ are $S$-invariant. Therefore both sides are zero, giving $S U x=U S x(x \in N)$, and hence $M \in$ Lat $S^{(2)}$.

A standard argument (cf. [9, Lemma 1]) now gives

Theorem 5. $A(\mathcal{S})$ is reflexive.

Given $A=\left[a_{i j}\right] \in \mathbb{M}_{n}(\mathrm{C})$, the $n \times n$ complex matrices, and $\mathbf{x}=\left(x_{1}, \cdots\right.$, $\left.x_{n}\right) \in X^{(n)}$, let $A \mathbf{x}$ denote the element $\mathbf{y}=\left(y_{1}, \cdots, y_{n}\right)$ in $X^{(n)}$ defined by

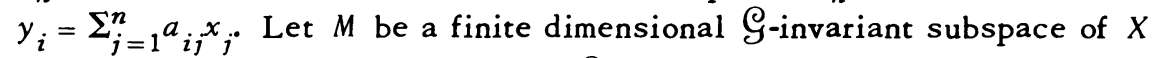

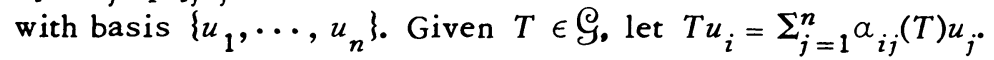

Lemma 6. The map $\alpha: T \rightarrow \alpha(T)=\left[\alpha_{i j}(T)\right]$ is an anti-representation of 乌 $\rightarrow M_{n}(\mathrm{C})$.

Define the operator $\mathbf{P}$ in $B\left(X^{(n)}\right)$ by

$$
\mathbf{P x}=\int_{\mathcal{S}} \alpha\left(T^{-1}\right) T^{(n)} \mathbf{x} d T
$$

where $d T$ denotes Haar measure on $\mathcal{G}$. $\mathbf{P}$ is a projection, but this fact is not needed here. What is needed is the following result, which is easily verified using Lemma 6.

Lemma 7. $\mathbf{P} T^{(n)} \mathbf{x}=\mathbf{P} \alpha(T) \mathbf{x}$ for $T \in \mathcal{G}$ and $\mathbf{x} \in X^{(n)}$.

Defining $\mathbf{u}$ in $X^{(n)}$ by $\mathbf{u}=\left(u_{1}, \cdots, u_{n}\right)$, we have $T^{(n)} \mathbf{u}=\alpha(T) \mathbf{u}$ for each $T \in \mathcal{G}$. Therefore, from the definition of $\mathbf{P}, \mathbf{P u}=\mathbf{u}$. Since $\mathbf{u} \neq \mathbf{0}$, it follows that ker $\mathbf{P}$ is strictly smaller than $X^{(n)}$. Thus, if $X^{*}$ is the dual space of $X$, there exists $\mathbf{f}=\left(f_{1}, \cdots, f_{n}\right) \in X^{*(n)}$ with $\mathbf{f} \neq \mathbf{0}$ such that $\mathbf{f}$ annihilates ker $\mathbf{P}$ (making the obvious identification of the dual space of $X^{(n)}$ with $\left.X^{*(n)}\right)$. Put

$$
F=\sum_{i=1}^{n} f_{i} \otimes u_{i}
$$

Then $F \neq 0$. Using the fact that $T^{(n)} \mathbf{x}-\alpha(T) \mathbf{x}$ belongs to ker $\mathbf{P}$ for every $T \in \mathcal{Y}$ and $\mathbf{x} \in X^{(n)}$, a routine calculation gives

Lemma 8. $F \in \mathfrak{S}^{\prime}$.

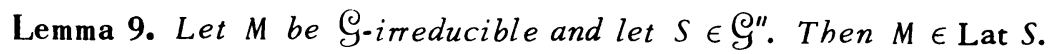


To see this observe that $F$ and $S$ commute. Further $\{0\} \neq F(X) \subset M$,

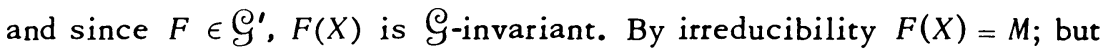
then $S(M)=S F(X)=F S(X) \subset M$.

Using reflexivity, Lemmas 9 and 3 give $\mathcal{Y}^{\prime \prime} \subset A(\mathcal{G})$. Since the reverse inclusion always holds, we have thus proved

Theorem 10. $乌^{\prime \prime}=A(乌)$.

Solution B. We give an example of a $G$-operator on a weakly sequentially complete space which is not of the form $\exp (i S)$ with $S$ bounded. This example depends on some general facts about logarithms of point measures.

Let $G$ be a LCAG and let $M(G)$ be the commutative Banach algebra of bounded regular complex measures on $G$ under convolution. Given $x \epsilon$ $G$, we show that the point mass $\delta_{x}$ has a logarithm in $M(G)$ if, and only if, $x$ is of finite order in $G$. The "if" proof follows from elementary spectral theory. For the converse, let $M_{d}(G)$ be the Banach algebra of discrete measures on $G$.

Lemma 11. If a discrete measure $\mu$ on $G$ has a logarithm in $M(G)$, then $\mu$ has a logarithm in $M_{d}(G)$.

This follows from the fact that if $\mu=\exp \nu$ for some $\nu \in M(G)$, then $\mu=\exp \nu_{d}$ where $\nu_{d}$ is the discrete part of $\nu$.

Lemma 12. Let $\delta_{x}=\exp \nu$ in $M(G)$. Then $x$ is of finite order in $G$.

By Lemma 11 we may (and do) assume that $G$ is discrete. Then the maximal ideal space of $M(G)$ is the compact group $\hat{G}$ dual to $G$. The proof can be completed by the following simple argument due to Gavin Brown.

Taking Gelfand transforms in the equation $\delta_{x}=\exp \nu$, we obtain

$$
x(\chi)=\hat{\delta}_{x}(\chi)=\exp \hat{\nu}(\chi) \quad(\chi \in \hat{G}),
$$

where, without loss of generality, $\hat{\nu}\left(\chi_{1}\right)=0$ for $\chi_{1}$ the unit of $\ddot{G}$. Since $x$ is a character on $\hat{G}$, it follows that

$$
\hat{\nu}(\chi \psi)=\hat{\nu}(\chi)+\hat{\nu}(\psi)+2 \pi i N(\chi, \psi) \quad(\chi, \psi \in \hat{G})
$$

where $N: \hat{G} \times \hat{G} \rightarrow \mathbf{Z}$ is continuous. Let $H$ be the connected component containing $\chi_{1}$ in $G^{-}$. Then $2 \pi i N(\chi, \psi)=-\hat{\nu}\left(\chi_{1}\right)=0$ on $H$. Hence

$$
\hat{\nu}\left(\chi^{n}\right)=n \hat{\nu}(\chi) \quad(\chi \in H, n \in \mathbf{Z}) .
$$

The boundedness of the continuous function $\hat{\nu}$ on the compact group $H$ 
gives $\hat{\nu}(\chi)=0(\chi \in H)$. Hence $x(\chi)=1(\chi \in H)$ and so $x$ is of finite order in $G[6,24.20]$.

We can now give the counterexample for Problem B. Let $R_{\omega}$ be the translation operator on $L^{1}(\mathbf{T})$ defined by

$$
\left(R_{\omega} f\right)(t)=f\left(t \omega^{-1}\right) \quad\left(f \in L^{1}(\mathbf{T}), t \text { a.e. }\right)
$$

where $\omega \in \mathbf{T}$ and $\arg \omega$ is an irrational multiple of $2 \pi \cdot R_{\omega}$ is a G-operator on $L^{1}(\mathrm{~T})$ [4, Example 5.4] and $L^{1}(\mathrm{~T})$ is weakly sequentially complete [3, IV. 8.6].

Theorem 13. $R_{\omega}$ does not have a logarithm in $B\left(L^{1}(\mathbf{T})\right)$.

For suppose $R_{\omega}=\exp S$ in $B\left(L^{1}(\mathrm{~T})\right)$. Since the powers of $\omega$ are dense in $\mathbf{T}$, it follows that $S$ commutes with every translation $R_{t}(t \in \mathbf{T})$. Hence $S$ is a multiplier on $L^{1}(\mathbf{T})$ and there exists $\mu \in M(\mathbf{T})$ such that $S f=\mu * f(f \epsilon$ $\left.L^{1}(\mathrm{~T})\right)[7$, Theorem 0.1 .1$]$. Therefore

$$
\delta_{\omega} * f=R_{\omega} f=(\exp \mu) * f \quad\left(f \in L^{\mathrm{l}}(\mathrm{T})\right)
$$

and so $\delta_{\omega}=\exp \mu$. Lemma 12 gives the required contradiction.

\section{REFERENCES}

1. K. de Leeuw, Linear spaces with a compact group of operators, Illinois $\mathrm{J}$. Math. 2 (1958), 367-377. MR 21 \#819.

2. K. de Leeuw and I. Glicksberg, Applications of almost periodic compactifications, Acta Math. 105 (1961), 63-97. MR 24 \# A1632.

3. N. Dunford and J. T. Schwartz, Linear operators. I: General theory, Pure and Appl. Math., vol. 7, Interscience, New York, 1958. MR 22 \# 8302.

4. T. A. Gillespie and T. T. West, Operators generating weakly compact groups, Indiana Univ. Math. J. 21 (1972), 671-688.

5. - Operators generating weakly compact groups. II, Proc. Royal Irish Acad. 73A (1973), 309-326.

6. E. Hewitt and K. A. Ross, Abstract harmonic analysis. Vol. 1: Structure of topological groups. Integration theory, group representations, Die Grundlehren der math. Wissenschaften, Band 115, Academic Press, New York; Springer-Verlag, Berlin, 1963. MR 28 \# 158.

7. R. Larsen, An introduction to the theory of multipliers, Die Grundlehren der math. Wissenschaften, Band 175, Springer-Verlag, Berlin, 1971.

8. Ju. I. Ljubič, Almost periodic functions in the spectral analysis of operators, Dokl. Akad. Nauk SSSR 132 (1960), 518-520 = Soviet Math. Dokl. 1 (1960), 593-595. MR 22 \# 9863.

9. H. Radjavi and P. Rosenthal, On invariant subspaces and reflexive algebras, Amer. J. Math. 91 (1969), 683-692. MR 40 \# 4796.

\footnotetext{
DEP ARTMENT OF MATHEMATICS, UNIVERSITY OF EDINBURGH, EDINBURGH, SCOTLAND
} 